Document downloaded from:

http://hdl.handle.net/10251/136477

This paper must be cited as:

Ramirez-Nunez, JA.; Antonino-Daviu, J.; Climente Alarcon, V.; Quijano-Lopez, A.; Razik, H.; Osornio-Rios, RA.; Romero-Troncoso, RJ. (2018). Evaluation of the detectability of electromechanical faults in induction motors via transient analysis of the stray flux. IEEE Transactions on Industry Applications. 54(5):4324-4332.

https://doi.org/10.1109/TIA.2018.2843371

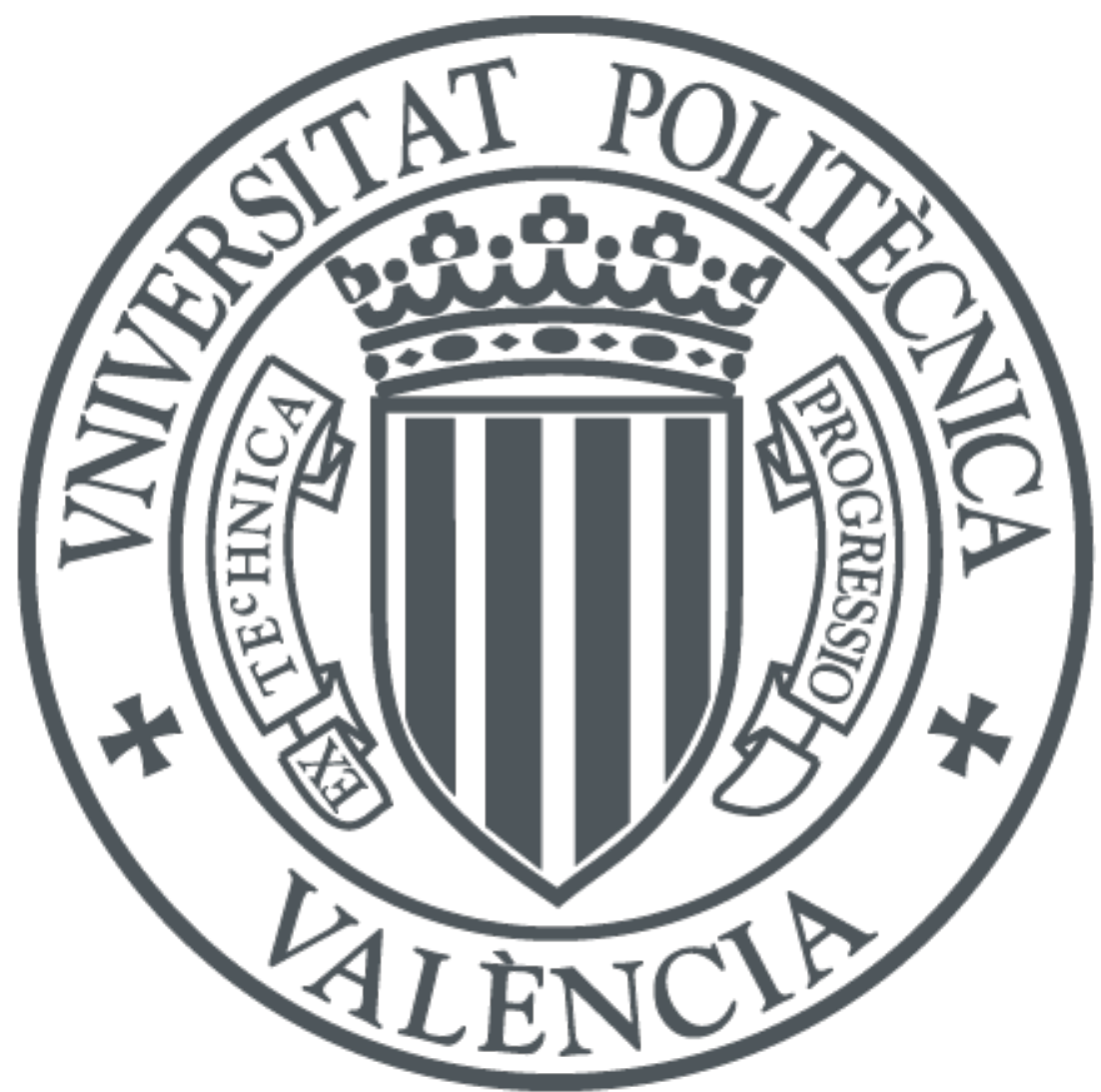

The final publication is available at

https://doi.org/10.1109/TIA.2018.2843371

Copyright Institute of Electrical and Electronics Engineers

Additional Information 


\title{
Evaluation of the detectability of electromechanical faults in induction motors via transient analysis of the stray flux
}

\author{
Juan A. Ramirez-Nunez**, Jose Antonino-Daviu*, IEEE Senior Member, Vicente Climente-Alarcón***, IEEE Member, \\ Alfredo Quijano-López*, Hubert Razik****, IEEE Senior Member, Roque A. Osornio-Rios**, IEEE Member, \\ Rene J. Romero-Troncoso**, IEEE Senior Member
}

\author{
*Instituto Tecnologico de la \\ Energia, Universitat \\ Politècnica de València, \\ Camino de Vera s/n, 46022, \\ Valencia, SPAIN \\ joanda@die.upv.es
}

\author{
** CA Mecatronica, \\ Facultad de Ingenieria, \\ Campus San Juan del Rio, \\ Universidad Autonoma de \\ Queretaro, San Juan del Rio \\ 76807, Queretaro, MEXICO
}

\author{
****Department of Electrical \\ Engineering and \\ Automation, \\ Aalto University, P. O. Box \\ 15500 \\ FI-00076 Aalto, FINLAND \\ viclial@ieee.org
}

\author{
**** Université de Lyon, \\ Ampère, CNRS UMR 5005, \\ Ecole Centrale de Lyon, \\ INSA-Lyon, UCB Lyon 1, \\ F-69100, Villeurbanne \\ FRANCE \\ Hubert.razik@univ-lyon1.fr
}

\begin{abstract}
The stray flux that is present in the vicinity of an induction motor is a very interesting information source to detect several types of failures in these machines. The analysis of this quantity can be employed, in some cases, as a supportive tool to complement the diagnosis provided by other quantities. In other cases, when no other motor quantities are available, stray flux analysis can become one of the few alternatives to evaluate the motor condition. Its non-invasive nature, low cost and easy implementation makes it a very interesting option that requires further investigation. The aim of this work is to evaluate the suitability of the stray flux analysis under the starting transient as a way to detect certain faults in induction motors (broken rotor bars and misalignments), even when these types of faults coexist in the motor. To this end, advanced signal processing tools will be applied. Several positions of the flux sensors are considered in this study. Also, for the first time, a fault indicator based on the stray flux analysis under the starting is introduced and its sensitivity is compared versus other indicators relying on other quantities. It must be emphasized that, since the capture of the transient and steady-state flux signals can be carried out in the same measurement, the application of the approach presented in this work is straightforward and its derived information may become crucial for the diagnosis of some faults.
\end{abstract}

Keywords-induction motor; fault diagnosis; transient analysis; wavelet transforms; rotor faults; misalignment

\section{INTRODUCTION}

Induction motors fault detection methods relying on the use of the external magnetic field have been known over decades. These methods rely on the fact that the presence of a fault in the motor modifies the magnetic field in the vicinity of the machine [1-3]. Hence, a proper analysis of this quantity with suitable tools can enable to identify possible evidences of the presence of the failure. Some authors have defended the use of the stray flux analysis as a valuable diagnosis tool, emphasizing its non-invasive nature and simplicity [1]. Moreover, there has been a progressive reduction of the cost and volumes of the flux sensors and today it is possible to find small and advanced sensors at very low prices [4]. Logically, the technique has its own constraints such as the difficult introduction of fault severity indicators or the influence of the sensor position over the results $[1,5]$. These are some reasons that justify its much more limited penetration in industry in comparison with other alternative techniques, such as current or vibration analysis. For instance, current analysis is being successfully employed in industry to diagnose certain faults such as rotor damages or eccentricities. The combination of classical approaches relying on the analysis of steady-state currents (MCSA) [6-7] with modern methods based on the startup current analysis (that avoid some limitations of the classical approaches) [8-10] is providing very reliable results for the diagnosis of the aforementioned failures. This fact is of special interest, considering that in a single measurement the user can capture both necessary signals (startup+ steady-state current) without necessity of additional sensors or equipment. Moreover, the monitoring of the motor current can be carried out remotely, from the Motor Control Center (MCC) or Panelboard [11]. However, there are applications where current analysis may not be conclusive due to a diversity of causes [6] or where its sensitivity for the detection of some faults may be improvable. In this context, the analysis of the stray flux may become an interesting complementary information source for the diagnosis. For instance, some current investigations are oriented to study the potential of the stray flux analysis to provide immunity to axial air duct influence and load oscillations [12]. Moreover the measurement of this quantity only requires the installation of a simple flux sensor in the external frame, which is typically based on a coil, where the induced electromotive force $e$ is monitored [1, 13]. Although some industrial users may be reluctant to install additional sensors, the necessary coil sensors are external to the machine and do not perturb its operation conditions; this may be an always preferable option than having eventual false indications, especially for large

This work was supported in part by the Spanish 'Ministerio de Economía y Competitividad' (MINECO) and FEDER program in the framework of the 'Proyectos I+D del Subprograma de Generación de Conocimiento, Programa Estatal de Fomento de la Investigación Científica y Técnica de Excelencia' (ref: DPI2014-52842-P)." 
machines, where the implications of these false diagnostics in terms of cost can amount for several million \$ [11] .

Although there have been several works proposing the use of the stray flux for fault diagnosis purposes, they are focused on the analysis at steady-state. Most of these works rely on the evaluation of certain components that are amplified by the corresponding fault in the FFT spectrum of the steady-state signal $[1,5,13]$. However, as far as the authors know, no works have dealt with the analysis under transient regime (e.g. under the starting): the only related references in this regard are [14] which is the initiation of the investigation culminated in this paper and [15] that preliminarily suggests transient analysis to detect short faults. Bearing in mind that the starting and steady-state flux signals can be captured in the same measurement, it is illogical to renounce to the potential information that the analysis of the flux under the starting can provide. Moreover, the previous experience with startup current analysis has shown that some false indications of the steady-state analysis (such as those caused by rotor axial ducts or rotor core magnetic anisotropy) can be avoided due to limited flux penetration into the rotor yoke during the startup due to cage eddy current rejection [9, 16]. Furthermore, in many applications the motors rarely operate under stable regimes so the application of methods based on the analysis of stationary flux signals may be difficult. Hence, it is worthy to explore the viability and sensitivity of the transient analysis of the stray flux for the diagnosis of electromechanical faults in induction motors.

This work is focused on the analysis of the stray flux under the motor starting. The objective is to apply advanced signal processing tools, which are being currently employed for the analysis of other transient quantities, in order to detect eventual evidences of the presence of the considered failures (rotor faults and misalignments), even when these failures are simultaneously present in the motor. In the paper, both continuous and discrete time-frequency transforms are employed. More specifically, an optimized version of the Short Time Fourier Transform (STFT) and the Discrete Wavelet Transform (DWT) will be used. It is shown that the presence of these failures leads to the appearance of characteristic patterns in the time-frequency analyses of the startup flux signals. These patterns can be used for the reliable diagnosis of these faults and, in some cases, they are even clearer than those appearing when analyzing other quantities. Three different flux sensors positions are considered in the study. Moreover, a fault severity indicator based on transient flux analysis is proposed. The analysis of its sensitivity shows that it can be comparable with the sensitivity provided by other indicators utilized in well-known techniques. The results confirm the potential of the transient analysis of the flux as a supportive information source that could be incorporated in induction motor predictive maintenance programs.

\section{ANALYSIS OF THE STRAY FLUX UNDER THE STARTING}

In previous works, it was proven that the analysis of the external magnetic field, measured with proper sensors, can enable to detect several types of failures in induction motors.
With regards to rotor faults, in [13] is proven that components at $s \cdot f$ and $3 \cdot s \cdot f$ (where $f=$ supply frequency and $s=$ slip) in the axial field are particularly sensitive to the presence of rotor damages. This latter component is due to the angular speed variation at $2 \cdot s \cdot f$ caused by the presence of broken bars [1, 13]. Monitoring the amplitudes of these components in the FFT spectrum of the external magnetic field (especially, in the axial direction) is an interesting option to detect rotor damages. However, the low frequency values of these components, makes it necessary to have long acquisition times, so that the FFT analysis is able to properly distinguish them $[1,13]$.

The authors of [13] also demonstrate that the amplitude of the aforementioned component at $s \cdot f$ can be also affected by the presence of dynamic eccentricities in the machine that can lead to the amplification of that component.

Besides, Bellini and other authors [5] proved the usefulness of the stray flux analysis by studying the sideband components, given by $f \pm 2 \cdot s \cdot f$, appearing around the fundamental component in the Fourier spectra of the electromotive force $(\mathrm{emf})$ induced in a coil sensor. As stated in [13], and unlike what happens with the components at $s \cdot f$ and $3 \cdot s \cdot f$ which have an axial nature, the components at $f \pm 2 \cdot s \cdot f$ are primarily present in the radial external magnetic field. This is particularly relevant since, depending on the flux sensor position, the predominant fault components in the induced emf can be the axial or the radial ones. In this regard, Fig. 1 shows the three flux sensor positions considered in the present paper. In agreement with [13], in Position A, the sensor measures the axial field. However, this position is not always easily accessible in industry. In Position B, the sensor measures the radial field but also a portion of the axial field. Finally, in Position C, the sensor predominantly measures the radial field. Depending on the sensor position, the radial or axial components will prevail in the transient analyses of the emf signals, which will be shown later.
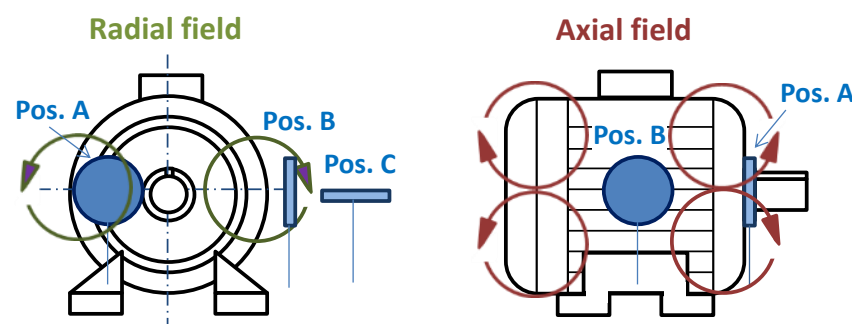

Fig.1 Considered coil sensor positions

On the other hand, some authors [6, 19] have stated that the presence of misalignments increases the amplitudes of components at $f \pm f_{r}$ ( $f_{r}=$ rotor rotational frequency) in the spectrum of the stator current. The amplitudes of these same components can be also amplified due to the possible presence of mixed eccentricities in the machine (i.e. combination of static and dynamic eccentricities). Other authors [17] state that mixed eccentricities lead to the amplification of similar frequencies in the FFT spectrum of the steady-state flux. 
These frequencies can be also written as (1) ( $p=$ pole pair number, $m=1,2,3 \ldots)$.

$$
f_{\text {ecc }}=f \cdot(1 \pm m \cdot(1-s) / p)
$$

The method presented in this paper relies on studying how the aforementioned components evolve under the starting and tracking them with suitable time-frequency tools. In this regard, the variation of the slip $s$ under a direct-on-line starting will lead to a particular variation of the aforementioned fluxrelated fault components (both of those associated with broken bars (axial and radial) and those linked with eccentricities/misalignments). More specifically, these components will evolve in a characteristic way, when the slip $s$ changes between 1 and near 0 during a startup (see Fig. 2):

- Firstly, in healthy conditions, only the fundamental component is expected at the low frequency region under analysis. In the time-frequency map, it will appear as a horizontal line (which may be thicker depending on the resolution of the employed time-frequency transform). Moreover, there are some additional harmonics that can partially evolve in that region, such as the slotting harmonics that were widely studied in works as [13]: the frequencies of some of these harmonics decrease to zero as the machine accelerates under starting. However, their evolutions in the low frequency region (below $f$ ) are usually much less noticeable than the fault-related components due to their small amplitudes and they are circumscribed to the initial instants of the starting after the connection.

- With regard to the broken bar-related harmonics, there are two main groups of components: the axial and radial ones. The axial component at $s \cdot f$ will drop from $50 \mathrm{~Hz}$ when the machine is connected $(s=1)$ to near $0 \mathrm{~Hz}$ at steady state $(s \approx 0)$, while the axial component at $3 \cdot s \cdot f$ will follow an analogue evolution but starting at $150 \mathrm{~Hz}$ and ending at near $0 \mathrm{~Hz}$. The frequency of the radial component at $f \cdot(1-2 \cdot s)$ will drop first to zero and then increase again until it reaches a value near $f$ at steadystate, following a characteristic V-shaped pattern that is similar to that followed by the lower sideband harmonic (LSH) present in the motor current [8]. The theoretical evolutions of all these components during a simulated transient are shown in Fig.2. Depending on the position of the flux sensor, a greater portion of axial or radial flux will be captured. As a consequence, the sensor position will determine a better distinction of the radial or axial components in the resulting analyses of the emf signals, as shown in this work.

- On the other hand, with regard to the mixed eccentricities or misalignments, for a machine with $p=2$, the two main fault components $(m=1)$ would start at the supply frequency $(50 \mathrm{~Hz})$ and would end at near $25 \mathrm{~Hz}\left(f_{\text {misal }}(-)\right)$ and $75 \mathrm{~Hz}\left(f_{\text {misal }}(+)\right)$ under the starting (see Fig.2). In previous works [18], it was found that when rotor faults and eccentricities coexist in the machine, the analysis of the startup current has problems to detect the eccentricity/misalignment fault due to the preponderance of the rotor fault signature in the results of the t-f analyses.

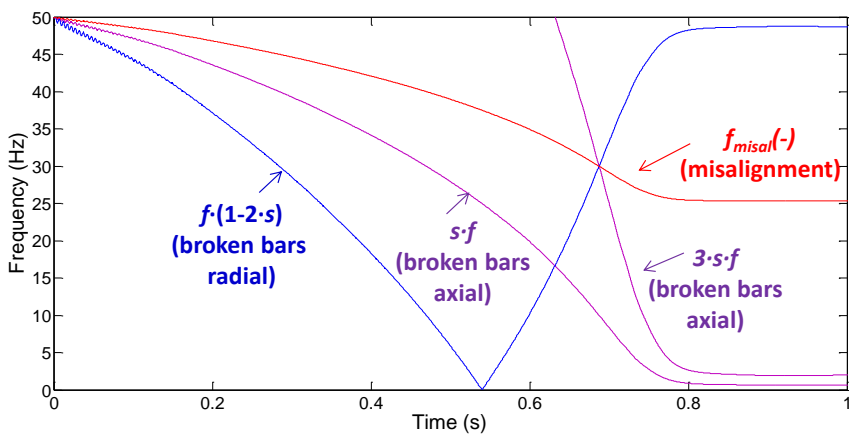

Fig. 2 Theoretical evolutions under the starting for the broken bar-related components (axial and radial) and for the misalignment components

The identification of the evolutions of all these components with the aid of proper time-frequency tools, such as the Discrete Wavelet Transform (DWT) or the Short-Time Fourier Transform (STFT), would constitute a reliable indicator of the presence of the corresponding fault. Moreover, it is possible to quantify the level of fault severity by evaluating the amplitudes of the components in the time-frequency maps resulting from the application of the aforementioned timefrequency tools.

\section{EXPERIMENTAL RESULTS IN LABORATORY MOTORS}

The method was validated in the lab on two motors with different characteristics: on the one hand, a SIEMENS 4 pole, $400 \mathrm{~V}, 1.1 \mathrm{~kW}$ cage induction motor with 28 bars (Motor 1) and, on the other hand, a WEG 6 pole, $400 \mathrm{~V}, 0.75 \mathrm{~kW}$ cage induction motor with 36 bars (Motor 2), where the two considered failures were forced (Fig. 3). On the one hand, the bar breakages where reproduced by drilling a hole in the connection point between the bar and the short-circuit end ring. In this particular study, only adjacent broken bars were considered. On the other hand, to force the misalignment condition, the motor was set at an angle to the driven machine in the horizontal direction (Fig. 4). As a result, an angular misalignment of $6^{\circ}$ was measured in the experiments. The flux sensor was manufactured in the laboratory and it was based on a coil of 1000 turns that was fixed to the external part of the motor frame. Its dimensions are specified in Fig.5. Three different sensor positions were tested (also depicted in Fig. 5) which correspond to the positions shown in Fig.1. Different tests were performed for each motor, under different fault and loading conditions. More specifically, three faulty cases where considered: healthy machine (with an inherent level of eccentricity), machine with misalignment and machine with misalignment + two broken bars. In each test, the emf signal delivered by the coil sensor was captured via a waveform recorder both under starting and at steady-state (40 seconds), using a sampling rate $f_{s}=5 \mathrm{kHz}$. These signals were later transmitted to a computer, where the corresponding analyses were performed. The Fast Fourier Transform (FFT) was employed for the stationary analyses while the optimized Short Time Fourier Transform (STFT) and the Discrete Wavelet Transform (DWT) were used for the analyses of the emf signals under the starting. For comparative purposes, the motor current waveform was registered in each of the tests (both under the starting and at steady-state). 


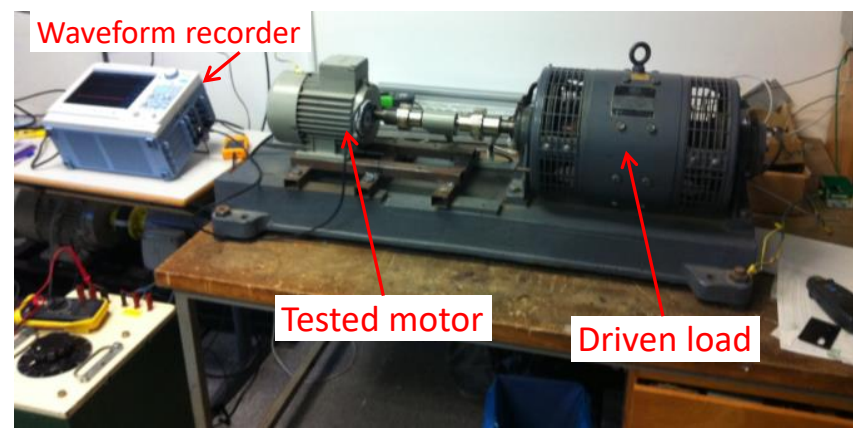

Fig. 3 Laboratory test bench

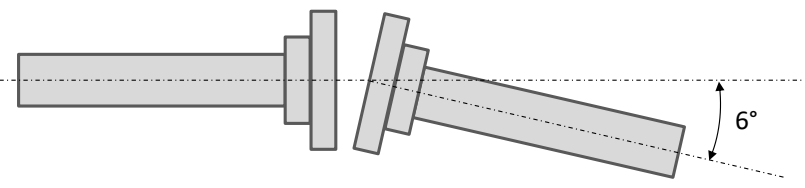

Fig. 4 Tested angular misalignment condition (horizontal direction)
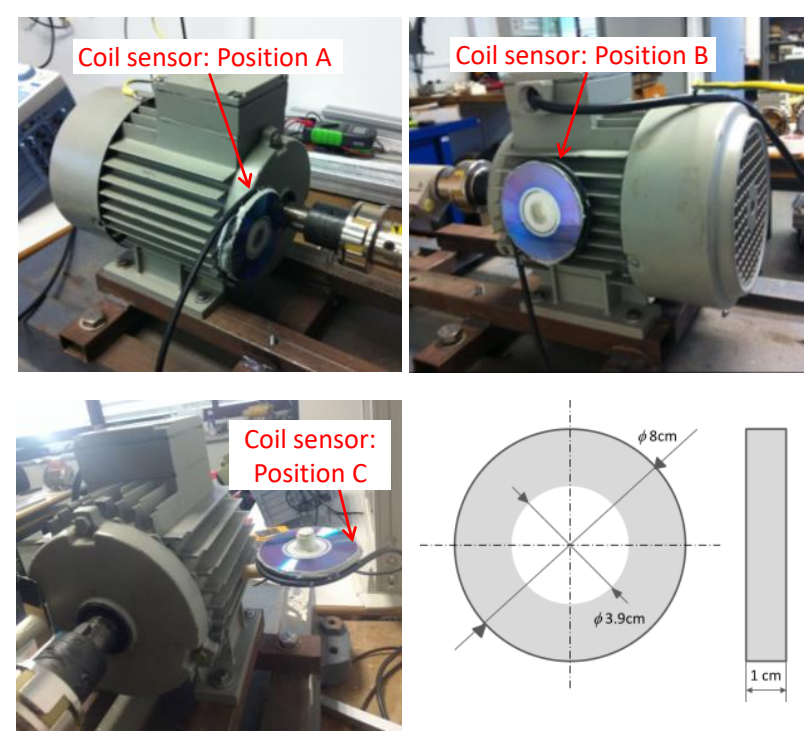

Fig. 5. Three tested positions and dimensions of the flux sensor.

Figure 6 shows the FFT analyses of the coil sensor emf signals at steady-state for Motor 1 , for the three considered faulty cases (healthy machine (with an inherent level of eccentricity), machine with misalignment and machine with misalignment + two broken bars) and for the three considered sensor positions. Note the higher amplitudes of the $s \cdot f$ and $3 \cdot s \cdot f$ components for the broken bar case for the sensor positions A and $\mathrm{B}$. On the contrary, at position $\mathrm{C}$, the components have much smaller amplitudes since, at that position, the flux captured by the sensor is mostly radial [13]. Note that when the misalignment is present, the component at $s \cdot f$ is discernible but has much more reduced amplitude than for the broken bar case. The amplitude of this component is slightly higher for the misalignment case, in comparison with the case in which only inherent eccentricity is present. This indicates certain dependence between the amplitude of that component and the level of misalignment, as stated in [13], although the influence of broken bars is much higher. On the other hand, note also the higher sensitivity at steady-state of the sensor at position B, in comparison with position $\mathrm{A}$, due to the larger portion of flux captured.

Regarding the transient analyses, as commented above, two different time-frequency tools were applied to the coil sensor signals captured during the startup of the motor: the STFT was employed to detect the presence of the fault components by identifying the characteristic $t-f$ evolutions of those components during the transient (which were described in the previous section). On the other hand, the DWT was utilized to develop a flux-based fault indicator that intends to indicate the rotor fault severity. This indicator has been defined by expression (2); it is analogue to the expression used for fault severity indicators relying on startup current analysis that were introduced in previous works [8]. It is well-known that the DWT performs a band pass-filtering of the analyzed signal (in this case, the transient emf). It decomposes the signal into a set of 'wavelet signals', each one covering a specific frequency range that is well-determined, since depends on the sampling rate that is used for capturing the emf signal [8]. The proposed indicator is based on the energy of one of these wavelet signals. More specifically, we propose using the signal $d_{8}$ that, considering a sampling rate of $5 \mathrm{kHz}$, would cover the frequency range $\sim[10 \mathrm{~Hz}-20 \mathrm{~Hz}]$. This signal has been selected since it covers part of the frequency band through which the $s \cdot f$ component evolves under starting; when this harmonic is present, the energy of this signal will increase, whereas it will remain low if the harmonic is absent. Hence, this can be used as a good indicator of the presence of the fault. Fig. 7 illustrates the difference between $d_{8}$ signal resulting from the DWT for a healthy motor and for a motor with broken bars. The proposed indicator, given by (2), relates the energy of the $d_{8}$ signal in the shadowed area (denominator of the logarithm in (2)) to the energy of the total emf signal in the same time interval (numerator of (2)) and expresses this ratio in $\mathrm{dB}$. In (2), emf is the value of the $j$ th sample of the emf signal; $d_{8}(j)$ is the $j$ element of the wavelet signal $d_{8} ; N_{b}$ is the number of the sample corresponding to the starting point of the group of oscillations caused by the $s \cdot f$ component in $d_{8} ; N_{s}$ is the number of sample at which these oscillations are extinguished (see Fig. 7). Note that, due to the dependence of the sensor location on the results, the idea of this indicator is to be computed always for the same position of the sensor in the diagnosed motor and to trend the value of this indicator over time.

Figure 8 shows the STFT analyses of the coil sensor signal during the startup for the Motor 1 under the three faulty conditions (sensor at positions A, B and C, as depicted in Fig.5). Interestingly, note that the startup flux analysis is able to detect the evolutions the different fault components (axial and radial). Their respective amplitudes depend on the corresponding position on the sensor: when the sensor is at position $\mathrm{A}$, the transient evolution of the $s \cdot f$ component is clearly visible (with frequency decreasing from 50 to $0 \mathrm{~Hz}$ ); it is already present in healthy condition (due to the inherent eccentricity in the machine) but its amplitude significantly increases when the broken bar fault is present. At position B, the same reasoning can be applied; the amplitude of that component is even higher for the broken bars case. On the contrary, at position $\mathrm{C}$, the evolution of the radial component at 
$f_{s b}=f \cdot(1-2 \cdot f)$ is significant and is clearly noticeable in the timefrequency map, overlapping the $s \cdot f$ evolution. This axial component is still present but it is much less evident that for the previous two previous sensor positions.

On the other hand, with regards to the misalignment detection, note that the $s \cdot f$ amplitude is affected by that fault. However, note that this component does not show an important increment when the misalignment level gets worse (compare the healthy case and that with misalignment in all cases). This seems to be coherent with the previous steady-state analyses. On the other hand, note that when the misalignment increases, the component $f_{\text {misal }}(-)$ is clearly noticeable in all cases. This component seems to be more sensitive than $s \cdot f$ for the detection of this fault and it would be an excellent indicator to compute the misalignment level in the machine.

For comparative purposes, Fig. 9 shows the analyses of the starting currents for the three considered cases (healthy machine (with an inherent level of eccentricity), machine with misalignment and machine with misalignment + two broken bars) for the Motor 1. Note that the starting current analysis is clearly able to detect the V-shaped pattern caused by the LSH due to broken rotor bars, which was well-characterized in previous works [8]. However, the detection of the misalignment pattern is not so easy. When only misalignment is present (see Fig. 9, middle), the misalignment component with frequency decreasing from $50 \mathrm{~Hz}$ to $25 \mathrm{~Hz}$ is still noticeable. However, when both faults coexist (Fig. 9, right), the broken bar pattern significantly masks the misalignment components evolution so that it is more difficult to detect that fault. This is coherent with the conclusions of other works [18]. In this context, the analysis of stray flux signals can provide interesting information for the diagnosis, in comparison with current analysis.

On the other hand, the computation of the DWT-based rotor fault indicator defined by (2), for Motor 1, is shown in Table I. The results included in the table show that the indicator experiences a small drop between healthy condition and the misalignment case for all sensor positions, while it experiences an important decrement when broken rotor bars are present. This indicates its especial suitability for rotor fault detection. On the other hand, note that the sensitivity is slightly better for the sensor at positions $\mathrm{A}$ and $\mathrm{C}$. Note, however, that at position $\mathrm{C}$ the values of the indicator are very high, due to the low amplitude of the fault component; this makes not advisable the use of this position for the computation of the indicator, since its value may be easily affected by any perturbation that is not related to the fault. In the table, the proposed flux-based indicator is compared with the indicator based on the startup current analysis that was introduced in previous works [8]. Note that for the detection of misalignments, the proposed flux indicator shows better results than the current indicator.

Finally, in order to confirm the generality of the method, Fig. 10 shows the STFT of the startup emf signals for the Motor 2 under the following conditions: 1) healthy state, 2) misalignment and 3) one broken bar plus inherent eccentricity. Note that analogue conclusions are reached in comparison with those obtained for Motor 1.

$$
\gamma_{D W T}(d B)=10 \cdot \log \left[\frac{\sum_{j=N b}^{N s} e m f_{j}^{2}}{\sum_{j=N b}^{N s}\left[d_{8}(j)\right]^{2}}\right]
$$
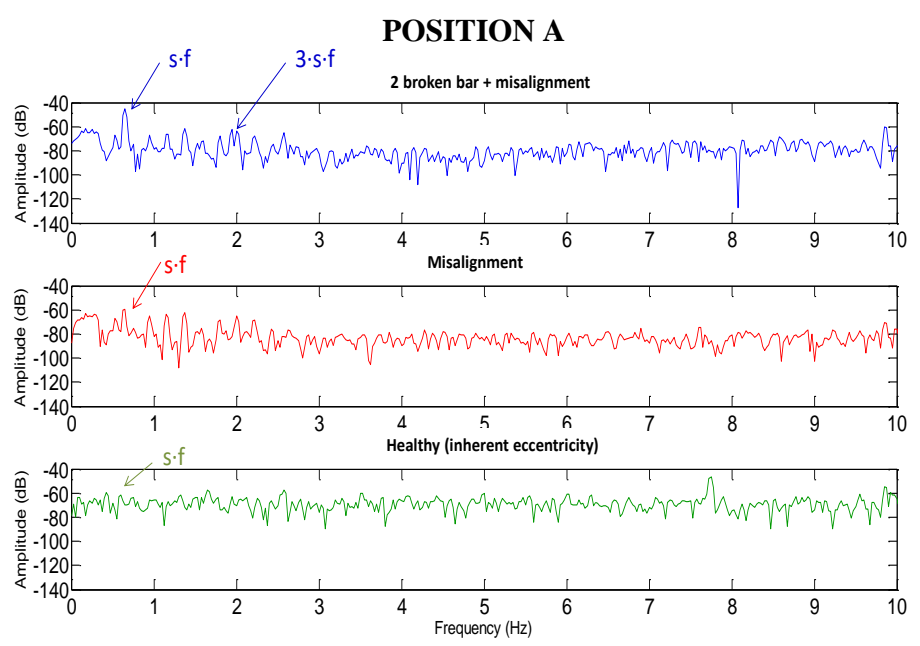

POSITION B
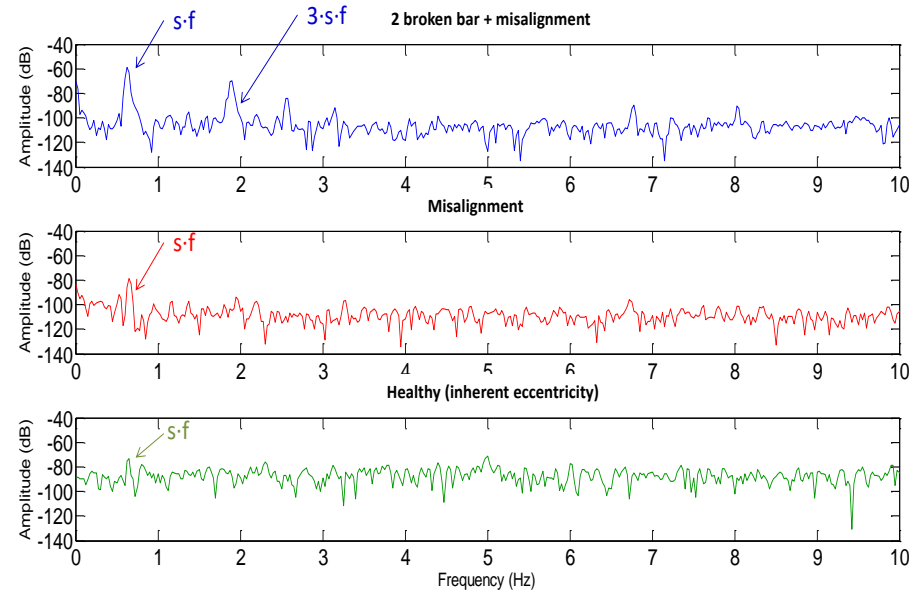

\section{POSITION C}
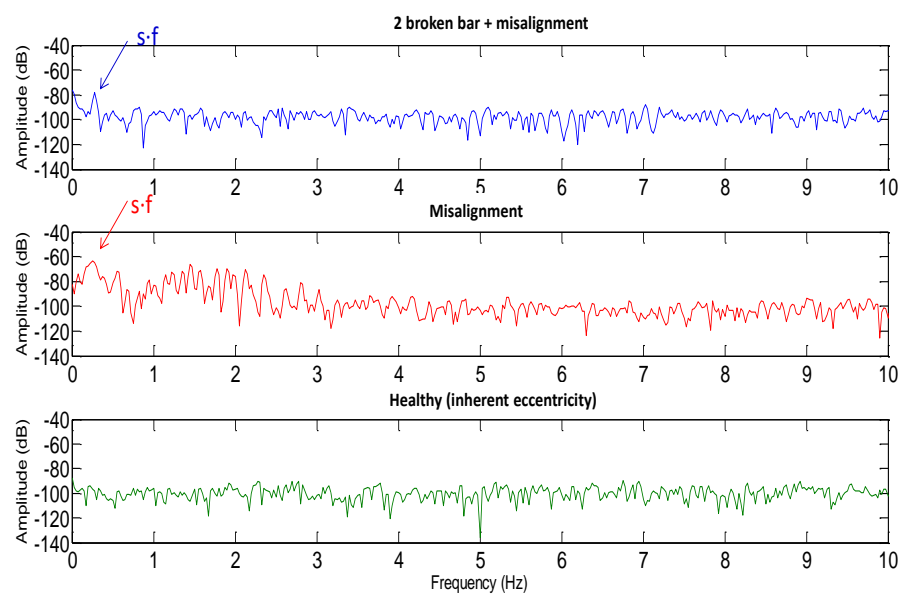

Fig. 6 FFT analyses of the coil sensor signal for the three faulty cases and for the three considered positions of the sensor (Motor 1). 

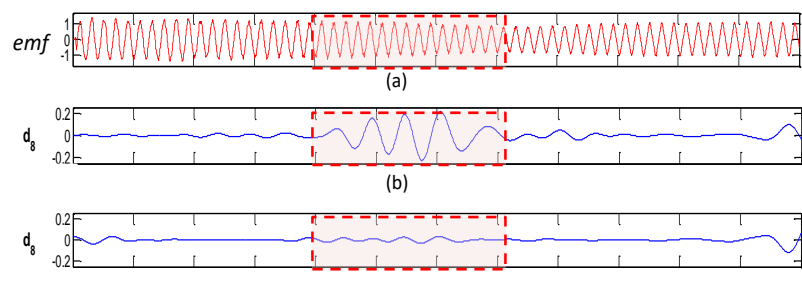

(c)

Fig. 7 DWT analysis of the emf signal (Motor 1): (a) total emf signal under starting, (b) signal $\mathrm{d}_{8}$ for a motor with rotor fault, (c) $\mathrm{d}_{8}$ signal for a healthy motor.
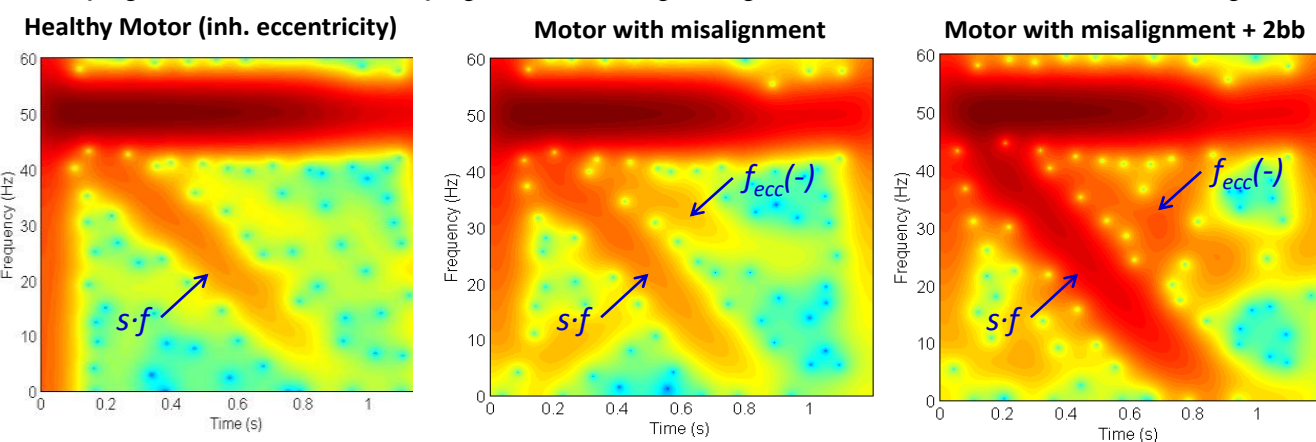

POSITION

\section{A}
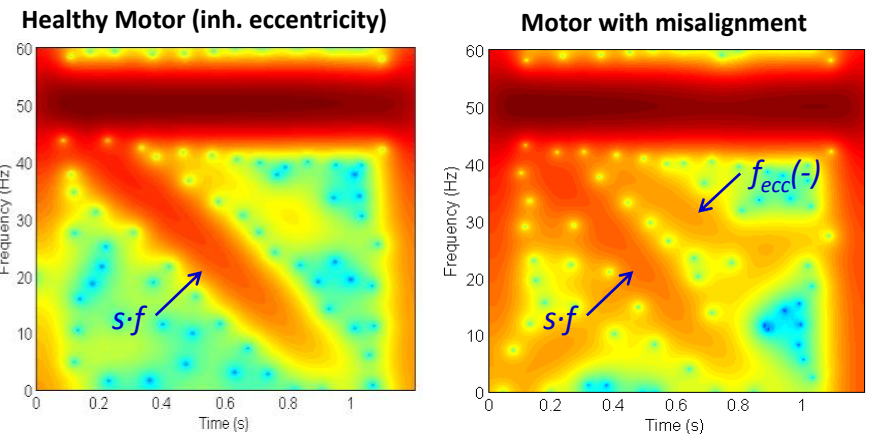

Motor with misalignment $+\mathbf{2 b b}$
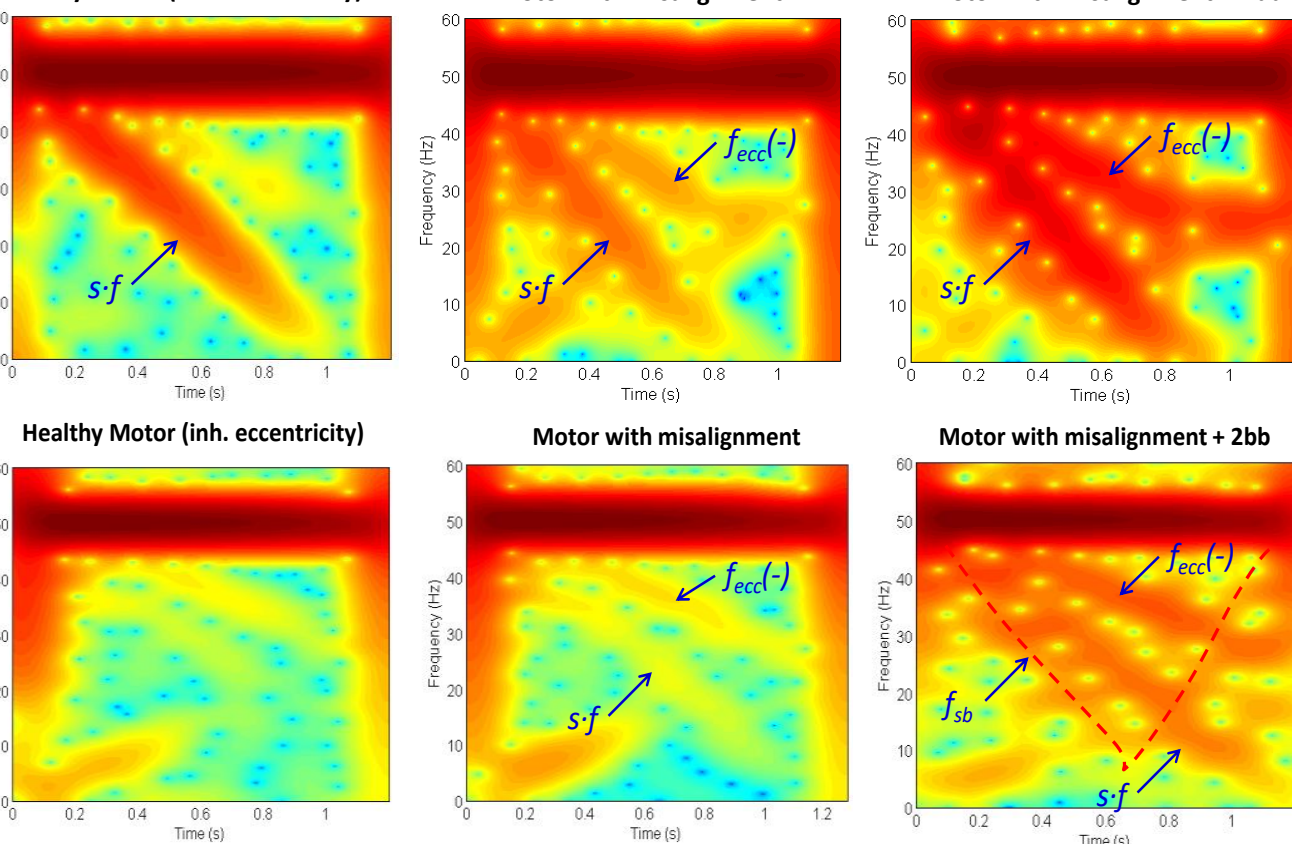

POSITION

B
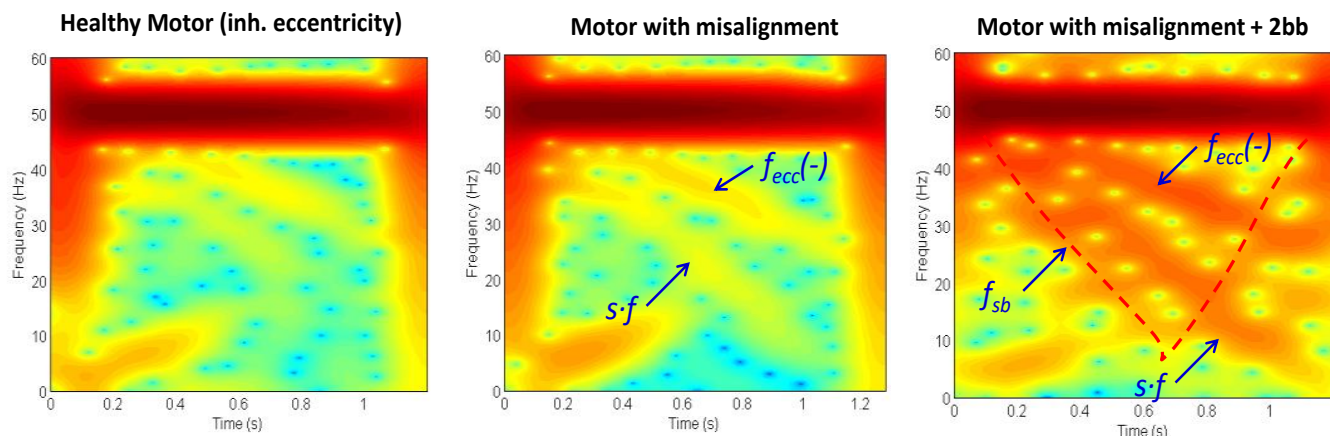

POSITION

Fig. 8 STFT analyses of the coil sensor signals for the machine (Motor 1) with inherent eccentricity, motor with misalignment and motor with misalignment + two broken bars and for the three considered positions of the sensor (the color denotes the energy density in each point of the time-frequency map, with red=highest density while blue=lowest density).
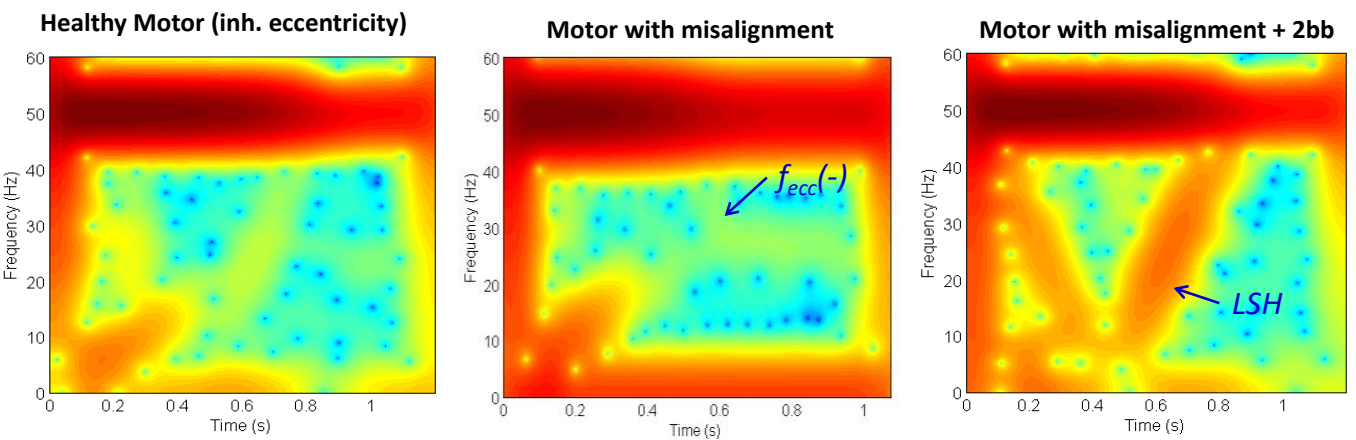

Fig. 9 STFT analyses of the starting current signals for the machine (Motor 1) with inherent eccentricity, motor with misalignment and motor with misalignment +two broken bars and for the three considered positions of the sensor (the color denotes the energy density in each point of the time-frequency map, with red=highest density while blue=lowest density). 

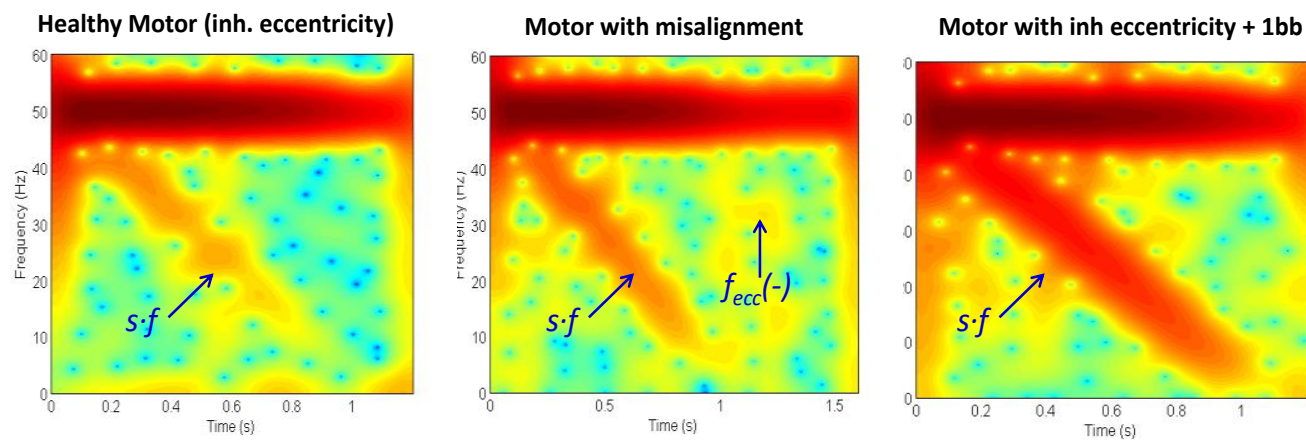

POSITION

A
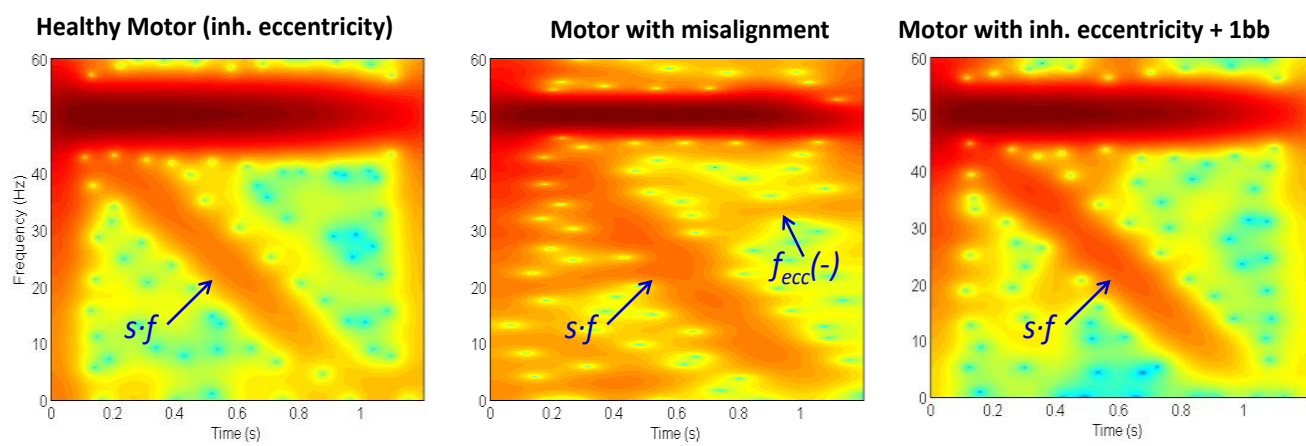

POSITION

B
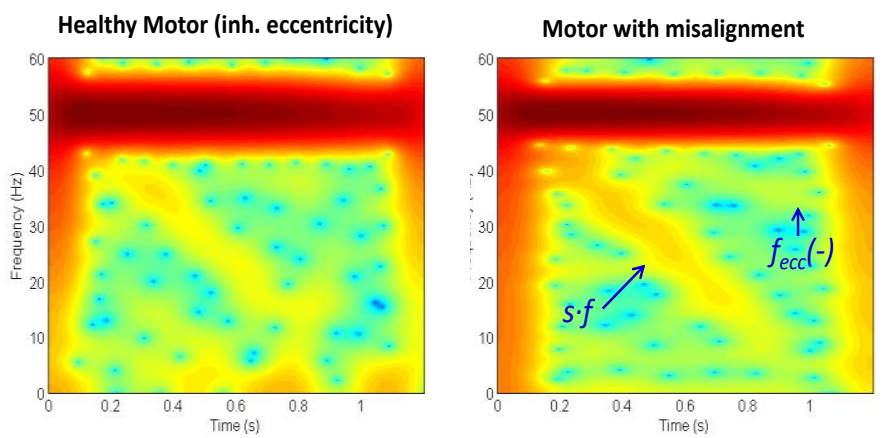

Motor with inh eccentricity $+1 b b$

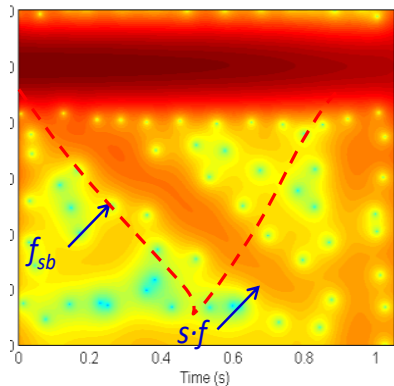

POSITION

C

Fig. 10 STFT analyses of the coil sensor signals for the machine (Motor 2) with inherent eccentricity, motor with misalignment and motor with inherent eccentricity +one broken bar and for the three considered positions of the sensor (the color denotes the energy density in each point of the time-frequency map, with red=highest density while blue=lowest density)

TABLE I. Rotor fault indicator (in $\mathrm{dB}$ ) based on DWT for the considered faulty cases and sensor positions (Motor 1). The respective difference versus healthy condition is specified between brackets.

\begin{tabular}{lccc} 
& $\begin{array}{c}\text { Healthy } \\
\text { (inherent ecc.) }\end{array}$ & Misalignment & $\begin{array}{c}\text { Misalignment + } \\
\text { broken bars }\end{array}$ \\
\hline $\begin{array}{l}\text { Flux indicator } \\
\text { pos. A }\end{array}$ & 38.1 & $34.1(-4 \mathrm{~dB})$ & $15(-23.1 \mathrm{~dB})$ \\
\hline $\begin{array}{l}\text { Flux indicator } \\
\text { pos. B }\end{array}$ & 33.3 & $27.18(-6.1 \mathrm{~dB})$ & $15.8(-17.5 \mathrm{~dB})$ \\
$\begin{array}{l}\text { Flux indicator } \\
\text { pos. C }\end{array}$ & 53.4 & $50.4(-3 \mathrm{~dB})$ & $30.8(-22.6 \mathrm{~dB})$ \\
\hline $\begin{array}{l}\text { Current } \\
\text { indicator }\end{array}$ & 51 & $48.2(-2.8 \mathrm{~dB})$ & $24(-27 \mathrm{~dB})$
\end{tabular}

\section{CONCLUSIONS}

This paper investigates, for the first time, the viability of the stray flux analysis under the starting to detect two types of failures in induction motors (rotor faults and misalignment), even when they coexist in the machine. Several signal processing tools are applied in the work for the analysis of the captured transient emf signals: on the one hand, a continuous tool, the STFT, is employed to track the evolutions of the fault harmonics during the startup. On the other hand, a discrete transform, the DWT, is used with the aims of computing a new rotor fault severity indicator relying on stray-flux analysis.

The results of the work prove that the advanced analysis of the startup flux can reliably diagnose the considered faults, even when they are simultaneously present in the machine. More specifically the following specific findings are obtained:

- Both considered faults seem to amplify the amplitude of the component at $s \cdot f$, despite it is much more 
sensitive to the broken bar fault. This idea is the basis to compute a rotor fault severity indicator based on the startup evolution of this component.

- The misalignment fault can be easily detectable with the component $f_{\text {misal }}(-)$ that is clearly amplified when the fault severity increases. Interestingly, this component is even observable when the broken bar fault is present. This is not so clear when analyzing startup currents, where the broken bar fault effects are usually much larger and often mask the misalignment components [18].

- The position of the flux sensor determines the type of components that can be visualized during the starting. While at Position A (and also B), the most noticeable components are the axial $(s \cdot f)$, at Position $\mathrm{C}$ the radial component evolutions (e.g. $f_{s b}$ ) are also relevant. This is important for the practical application of the method.

- Despite the smaller flux portion captured by the sensor when it is at Position $\mathrm{A}$ or $\mathrm{C}$, the rotor fault indicator at these positions seems to be more sensitive than when it is placed at Position B. In any case, the visualization of the transient evolutions of the fault components is clearer for this latter position of the sensor.

- The developed indicator based on startup flux analysis has a sensitivity that is comparable to that based on startup current analysis, which was developed in previous works.

An additional interesting conclusion of the investigation relies on the nature of the components caused by the misalignment condition considered in the paper $\left(f \pm f_{r}\right)$. The time-frequency analyses of the startup emf signals together with FFT analysis of steady-state emf signals seem to confirm that these components have a radial nature since their amplitudes suffer more prominent increments when the coil sensor is at Positions $\mathrm{B}$ and $\mathrm{C}$ rather than when it is at Position A.

In conclusion, the results prove that the analysis of the stray flux under starting can be employed as an effective supportive technique to complement the diagnosis provided by other quantities and may be a tool of potential interest for the field.

\section{REFERENCES}

[1] A. Ceban, R. Pusca and R. Romary, "Study of Rotor Faults in Induction Motors Using External Magnetic Field Analysis", IEEE Trans. Ind. Electronics, vol. 59, no. 5, pp. 2082-2093, 2012.

[2] H.Henao, C.Demian, G.A.Capolino, "A Frequency Domain Detection of Faults In Induction Machines Using an External Flux Sensor", IEEE Trans. Ind. Appl., Vol.39, No.5, Sept./Oct.2003, pp.1272-1279.

[3] H.Henao, G.A.Capolino, C.Martis "On the stray flux analysis for the detection of the three-phase induction machine faults", IEEE-IAS'03, St. Lake City, Oct. 2003.

[4] C. Jiang, S. Li and T. G. Habetler, "A review of condition monitoring of induction motors based on stray flux," 2017 IEEE Energy Conversion Congress and Exposition (ECCE), Cincinnati, OH, 2017, pp. 5424-5430.

[5] A. Bellini, C. Concari, G. Franceschini, C. Tassoni and A. Toscani, "Vibrations, currents and stray flux signals to asses induction motors rotor conditions", IECON 2006 - 32nd Annual Conference on IEEE Industrial Electronics, Paris, 2006, pp. 4963-4968.
[6] W.T. Thomson, I. Culbert, "Current Signature Analysis for Condition Monitoring of Cage Induction Motors", IEEE Press, Wiley, New Jersey, 2017.

[7] A. Bellini, F. Filippetti, G. Franceschini, C. Tassoni, R. Passaglia, M. Saottini, G. Tontini, M. Giovannini and A. Rossi, "On-field experience with on-line diagnosis of large induction motors cage failures using MCSA," IEEE Trans. on Ind. Appl., pp. 1045-1053, vol. 38, no. 4, July/Aug. 2002

[8] J. A. Antonino-Daviu M. Riera-Guasp, J. R. Folch, and M. Pilar Molina Palomares, "Validation of a new method for the diagnosis of rotor bar failures via wavelet transform in industrial induction machines," IEEE Trans. Ind. Appl., vol. 42, pp. 990-996, 2006.

[9] C. Yang, T-J. Kang, D. Hyun, S. Lee, J. Antonino-Daviu, J. PonsLlinares, "Reliable Detection of Induction Motor Rotor Faults Under the Rotor Axial Air Duct Influence," IEEE Trans. Ind. Appl., vol. 50, no. 4, pp. 2493-2502, Jul.-Aug. 2014.

[10] Jose Antonino-Daviu, Martin Riera-Guasp, Joan Pons-Llinares, Jongbin Park, Sang Bin Lee, Jiyoon Yoo and Christian Kral, "Detection of Broken Outer Cage Bars for Double Cage Induction Motors under the Startup Transient", IEEE Transactions on Industry Applications, vol. 48, no.5, pp. 1539-1548, Sept-Oct. 2012.

[11] S.B. Lee, E. Wiedenbrug., K. Younsi, "ECCE 2013 Tutorial: Testing and Diagnostics of Induction Machines in an Industrial Environment", presented at ECCE 2013, Denver, CO, USA, Sep 2013.

[12] L. Frosini, A. Albini and F. Beccarisi, "Detection of torque oscillations in induction motor drives by linear discriminant analysis," 2017 IEEE 11th International Symposium on Diagnostics for Electrical Machines, Power Electronics and Drives (SDEMPED), Tinos, 2017, pp. 510-516.

[13] R. Romary et al. "Electrical Machines Fault Diagnosis by Stray Flux Analysis" 2013 IEEE Workshop on Electrical Machines Design, Control and Diagnosis (WEMDCD), Paris, 2013, pp. 247-256.

[14] J. Antonino-Daviu, A. Quijano-López, V. Climente-Alarcon and H. Razik, "Evaluation of the detectability of rotor faults and eccentricities in induction motors via transient analysis of the stray flux," 2017 IEEE Energy Conversion Congress and Exposition (ECCE), Cincinnati, OH, 2017, pp. 3559-3564.

[15] H. Cherif, A. Menacer, R. Romary and R. Pusca, "Dispersion field analysis using discrete wavelet transform for inter-turn stator fault detection in induction motors," 2017 IEEE 11th International Symposium on Diagnostics for Electrical Machines, Power Electronics and Drives (SDEMPED), Tinos, 2017, pp. 104-109.

[16] J. A. Antonino-Daviu, J. Pons-Llinares, Sungsik Shin, Kun Wang Lee and Sang Bin Lee, "Reliable detection of induction motor rotor faults under the influence of rotor core magnetic anisotropy," in Proc.of the 2015 IEEE 10th International Symposium on Diagnostics for Electrical Machines, Power Electronics and Drives (SDEMPED), Guarda, 2015, pp. 14-21.

[17] L. Ishkova and O. Vitek, "Detection and classification of faults in induction motor by means of motor current signature analysis and stray flux monitoring", Przeglad Elektrotechniczny, R.92,NR 4/2016.

[18] J. Antonino-Daviu, P. Jover Rodriguez, M. Riera-Guasp, M. PinedaSánchez,A. Arkkio, "Detection of Combined Faults in Induction Machines with Stator Parallel Branches through the DWT of the startup current", Mechanical Systems and Signal Processing, Elsevier, vol. 23, no. 7, pp. 2336-2351, Oct. 2009.

[19] C. Verucchi, J.M. Bossio, G.B. Bossio, G. Acosta, "Misalignment detection in induction motors with flexible coupling by means of estimated torque analysis and MCSA," Mechanical Systems and Signal Processing, Vol. 80, Dec 2016, pp.570-581. 


\section{BIOGRAPHIES}

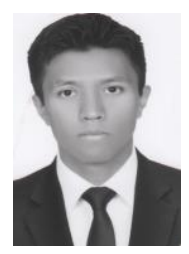

Juan A. Ramirez-Nunez received his M.S. degree on mechatronic from the Autonomous University of Queretaro, Mexico, in 2014. Currently he is working towards his Ph.D. at the Autonomous University of Queretaro, Mexico. He is part of the HSPdigital research group and his research interest include digital system, image processing, thermographic analysis for engineering applications and hardware signal processing on FPGA.

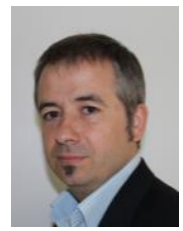

Jose A. Antonino-Daviu (SM'12) received his M.S. and Ph. D. degrees in Electrical Engineering, both from the Universitat Politècnica de València, in 2000 and 2006 , respectively. He also received his Bs. in Business Administration from Universitat de Valencia in 2012. He was working for IBM during 2 years, being involved in several international projects. Currently, he is Associate Professor in the Department of Electrical Engineering of the mentioned University, where he develops his docent and research work. He has been invited professor in Helsinki University of Technology (Finland) in 2005 and 2007, Michigan State University (USA) in 2010, Korea University (Korea) in 2014 and Université Claude Bernard Lyon 1 (France) in 2015. He is IEEE Senior Member since 2012 and he has published over 160 contributions, including international journals, conferences and books. He is Associate Editor of IEEE transactions on Industrial Informatics and acted as General Co-Chair of IEEE SDEMPED 2013.

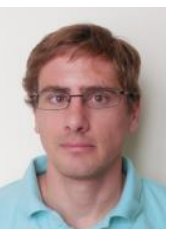

Vicente Climente-Alarcon (S'11-M'12) received the M.Sc. degrees in Chemical and Industrial Engineering in 2000 and 2011, and the Ph.D. degree in Electrical Engineering in 2012, all from the Universitat Politècnica de València (Spain). He worked as Adjunct Professor in the School of Industrial Engineering of the mentioned university, on research tasks in the area of condition monitoring of electrical machines and externally as a consultant in automation and management of power systems. In 2013 and from 2014 to 2017 he was with the Department of Electrical Engineering and Automation, Aalto University, Espoo, Finland, involved in research in the area of multiphysics simulations as visiting scholar, Postdoctoral Researcher and Docent. Currently he is a Research Associate at the Department of Materials Science and Metallurgy, University of Cambridge, Cambridge, UK, working on technological applications of superconductivity.

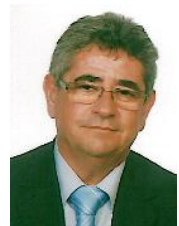

Alfredo Quijano-Lopez was born in 1960 in Valencia, Spain. He received the Electrical Engineer degree and the $\mathrm{Ph} . \mathrm{D}$. degree from Universitat Politècnica de València, in 1986 and 1992, respectively. He is the Head of the Instituto Tecnológico de la Energía. He is also a professor and a researcher at the Universitat Politècnica de València in the Electrical Engineering Department. His current research activity is focused on applied research for the Energy area and the electrical technology including renewable energies, high voltage, metrology, new materials and applications and research results transfer to companies.

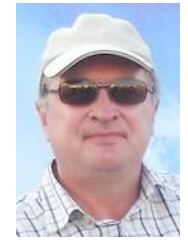

Hubert Razik (M'98-SM'03) received the degree from the Ecole Normale Supérieure, Cachan, France, in 1987, the Ph.D. degree in electrical engineering from the Institut Polytechnique de Lorraine, Nancy, France, in 1991, and the Habilitation to Supervise Researches from the Université Henri Poincaré, Nancy, in 2000. On November 1, 2009, he is currently a Full Professor of electrical engineering with Université Claude Bernard Lyon I, Villeurbanne, France. He is with the Laboratory Ampère-UMR 5005. Since January 1, 2016, he is a Deputy Director of the Laboratory AMPERE-UMR 5005 (www.ampere-lab.fr), Villeurbanne, France. Since July, 2016, he is the coordinator of the Master EEE-A (Electronic, Electrical Energy, Automatic) of the University Claude Bernard Lyon 1. His research interests include modeling, control, and monitoring conditions.

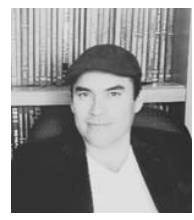

Roque A. Osornio-Rios (M'10) received the Ph.D degree in mechatronics from the Autonomous University of Queretaro, Queretaro, Mexico, in 2007. Dr. OsornioRios is a National Researcher level 2 with the Mexican Council of Science and Technology, CONACYT. He is currently a Head Professor with the University of Queretaro, Mexico. He is advisor for more than 80 theses, and a coauthor of more than 90 technical papers published in international journals and conferences. His fields of interest include hardware signal processing and mechatronics. Dr. Osornio-Rios is fellow of the Mexican Academy of Engineering. He is part of the editorial board of Journal and Scientific and Industrial Research

Rene de J. Romero-Troncoso (M'07-SM'12) received the Ph.D. degree in mechatronics from the Autonomous University of

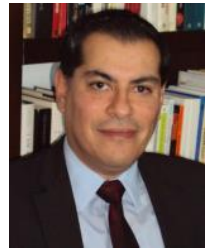
Queretaro, Mexico, in 2004. He is a National Researcher level 3 with the Mexican Council of Science and Technology, CONACYT, and Fellow of the Mexican Academy of Engineering. He is currently a Ful Professor with the Autonomous University of Queretaro, Mexico, and he has been an Invited Researcher with the University of Valladolid, Spain. He is author of two books on digital systems (in Spanish), and a coauthor of more than 170 technical papers published in international journals and conferences. His fields of interest include hardware signal processing with FPGA and monitoring and diagnosis on dynamic systems. Dr. Romero-Troncoso was a recipient of the 2004 Asociación Mexicana de Directivos de la Investigación Aplicada y el Desarrollo Tecnológico Nacional Award on Innovation for his work in applied mechatronics, and the 2005 IEEE ReConFig Award for his work in digital systems. 